\title{
Full counting-statistics in a single-electron transistor in the presence of nonequilibrium quantum fluctuations of charge
}

\author{
Yasuhiro Utsumi \\ Institut für Theoretische Festköperphysik, Universität Karlsruhe, 76128 Karlsruhe, Germany
}

(Dated: November 13, 2018)

\begin{abstract}
Using the Schwinger-Keldysh approach and the drone (Majorana) fermion representation, we evaluate current distribution in a single-electron transistor in a regime where a total tunnel resistance is small. Nonequilibrium quantum fluctuations of charge induce a lifetime broadening of chargestate level, as well as renormalization of system parameters. We find that the lifetime broadening effect may suppress the probability of relatively large current.
\end{abstract}

PACS numbers: $73.23 . \mathrm{Hk}, 72.70 .+\mathrm{m}$

Out of equilibrium, properties of carrier fluctuations at zero temperature, $T=0$, is beyond the scope of the 'fluctuation-dissipation theorem'. They have been a highlight of the mesoscopic quantum transport. Recently, the 'full counting statistics' (FCS) has pervaded as a powerful concept for a description of nonequilibrium current fluctuations [1]. The FCS is attractive because it would promote our understanding on nonequilibrium strongly-correlated mesoscopic systems. However only several attempts have been done for now [2, 3, 4].

A basic example of the strongly-correlated mesoscopic systems is a 'single-electron transistor' (SET), a metallic island coupled to left, right and gate electrodes with small total capacitance $C_{\Sigma}=C_{\mathrm{L}}+C_{\mathrm{R}}+C_{\mathrm{G}}$. The island can also exchange electrons with left (drain) and right (source) electrodes through tunnel junctions with resistance $R_{\mathrm{L}}$ and $R_{\mathrm{R}}$. However, the charging energy $E_{C}=e^{2} /\left(2 C_{\Sigma}\right)$ of a single electron can exceed source-drain bias voltage $V(e V>0)$ and insulate the island [Coulomb blockade $(\mathrm{CB})]$. One can reduce energy for impinging an electron into a charge-neutral island $\Delta_{0}=E_{C}\left(1-2 Q_{\mathrm{G}} / e\right)$ by gate-induced charge $Q_{\mathrm{G}}$. Then electrons start to tunnel through the island one by one [sequential tunneling (ST)], when $\Delta_{0}$ reaches a condition $\mu_{\mathrm{R}}<\Delta_{0}<\mu_{\mathrm{L}}$, where $\mu_{\mathrm{L} / \mathrm{R}}=\kappa_{\mathrm{L} / \mathrm{R}} e V\left[\kappa_{\mathrm{L} / \mathrm{R}}= \pm C_{\mathrm{R} / \mathrm{L}}\left(C_{\mathrm{L}}+C_{\mathrm{R}}\right)^{-1}\right]$ is a voltage drop between the $\mathrm{L} / \mathrm{R}$ electrode and the island.

The physics characteristic for strong electroncorrelations emerges when a total resistance $R_{\mathrm{T}}=$ $R_{\mathrm{L}} R_{\mathrm{R}} /\left(R_{\mathrm{L}}+R_{\mathrm{R}}\right)$ reduces to the order of the resistance quantum $R_{\mathrm{K}}=h / e^{2}$ and quantum fluctuations of charge are promoted 5]. The prime result is renormalization of the charging energy and the conductance. In the weak tunneling regime where the dimensionless conductance $\alpha_{0} \equiv R_{\mathrm{K}} /\left(4 \pi^{2} R_{\mathrm{T}}\right)$ is smaller than unity $\alpha_{0} \ll 1$, the scaling analysis predicted that the renormalization factor $z_{0}$ logarithmically depends on cutoff energy $\Lambda$, which is $T$ or $\mathrm{eV}\left(\hbar=k_{\mathrm{B}}=1\right)$, as $z_{0}=1 /\left\{1+2 \alpha_{0} \ln \left(E_{C} / \Lambda\right)\right\}[$ ] $]$.

For SET-like geometry, the FCS was discussed for an open quantum dot coupled to electrodes by singlechannel point contacts [3] and for a quantum dot in the Kondo regime [4]. However, for the metallic island, the FCS was obtained only in the limit of $\alpha_{0} \rightarrow 0$ [7]. The
FCS in the regime where the total resistance is small is still unknown.

In this paper, we evaluate the FCS of SET in the weak tunneling regime non-perturbatively using the Schwinger-Keldysh approach and the 'drone' (Majorana) fermion representation [8]. We will show out of equilibrium, strong quantum charge-fluctuations induce a lifetime broadening of charge-state level and suppress the probability for relatively large current to flow during a measurement. Hereafter, we assume the energy and spin relaxation times in each region are fast enough and electrons obey the Fermi distribution $f^{+}(\omega)=1 /\left(\mathrm{e}^{\omega / T}+1\right)$.

FCS in the weak tunneling regime. - In this regime, inverse of the $R C$ time $1 / \tau=4 \pi \alpha_{0} E_{C}=1 /\left(R_{\mathrm{T}} C_{\Sigma}\right)$ is smaller than $E_{C}$. It ensures that charge-state levels are well resolved and the low-energy physics is dominated by charge states with charge neutrality and with one excess electron. Then a Hamiltonian is mapped onto the 'multichannel anisotropic Kondo model' [a], by introducing a spin- $1 / 2$ operator $\hat{\sigma}$ acting on the two charge states,

$$
\begin{aligned}
& \hat{H}=\sum_{\mathrm{r}=\mathrm{L}, \mathrm{R}, \mathrm{I}} \sum_{k n} \varepsilon_{\mathrm{r} k} \hat{a}_{\mathrm{r} k n}^{\dagger} \hat{a}_{\mathrm{r} k n}+\frac{\Delta_{0}}{2} \hat{\sigma}_{z} \\
& +\sum_{\mathrm{r}=\mathrm{L}, \mathrm{R}} \sum_{k k^{\prime} n}\left(T_{\mathrm{r}} \hat{a}_{\mathrm{I} k n}^{\dagger} \hat{a}_{\mathrm{r} k^{\prime} n} \hat{\sigma}_{+}+\text {H.c. }\right),
\end{aligned}
$$

where $\hat{a}_{\mathrm{r} k n}$ annihilates an electron with wave vector $k$ in the left or right electrode $(\mathrm{r}=\mathrm{L}, \mathrm{R})$ or the island $(\mathrm{r}=\mathrm{I})$. We assumed the tunneling matrix element $T_{\mathrm{r}}$ is independent of $k$ and the index of channels for transverse mode and spin $n$. The microscopic parameters and the resistance of the junction $\mathrm{r}$ are related with each other as $R_{\mathrm{K}} / R_{\mathrm{r}}=$ $(2 \pi)^{2} N_{\mathrm{ch}}\left|T_{\mathrm{r}}\right|^{2} \rho_{\mathrm{I}} \rho_{\mathrm{r}}$, where $N_{\mathrm{ch}}$ is the number of channels. The electron density of states in each region $\rho_{\mathrm{r}}$ is assumed to be constant.

In the nonequilibrium state, the logarithmic renormalization of the system parameters can be treated by the 'resonant tunneling approximation' 9] but only for an average current. A technical difficulty arises because the spin-1/2 operator prevents to utilize Wick's theorem. A convenient device to avoid the problem might be the Majorana fermion representation [10], $\hat{\sigma}_{+}=\hat{c}^{\dagger} \hat{\phi}, \hat{\sigma}_{z}=2 \hat{c}^{\dagger} \hat{c}-1$ 
where $\hat{\phi}=\hat{d}^{\dagger}+\hat{d}$ is a Majorana fermion and $\hat{c}$ and $\hat{d}$ are Dirac fermions. The device enables one to utilize the fermionic Schwinger-Keldysh approach 11, 12.

A central quantity of the technique is the generating functional of connected Green function (GF) 13.

$$
W[\varphi] \equiv-i \ln \int D\left[a_{\mathrm{r} k n}^{*}, a_{\mathrm{r} k n}, c^{*}, c, d^{*}, d\right] \exp \left(i \int_{C} d t \mathcal{L}(t)\right),
$$

where $\mathcal{L}$ is the Lagrangian for $\hat{H}$ and we introduced six Grassmann variables. The closed time-path $C$ advances from $t=-\infty$ to $\infty$ and returns to $-\infty$. Then it connects to the imaginary time path and closes at $t=-\infty-i / T$. We introduce an auxiliary source field $\varphi(t)$, the phase of the tunneling matrix element $T_{\mathrm{r}} \rightarrow T_{\mathrm{r}} \mathrm{e}^{i \kappa_{\mathrm{r}} \varphi(t)}$, and assume a part for tunneling is switched on adiabatically. Now we can define fields on forward and backward branches $\varphi_{+}(t)$ and $\varphi_{-}(t)$, but only the center-of-mass coordinate $\varphi_{c}(t) \equiv\left\{\varphi_{+}(t)+\varphi_{-}(t)\right\} / 2$ has physical meaning and is fixed as $e V t$.

In the scheme of FCS, during measurement time $t_{0}$, the relative coordinate $\varphi_{\Delta}(t) \equiv \varphi_{+}(t)-\varphi_{-}(t)$ is switched on and is fixed as a constant $\lambda$ called the 'counting field' 12]:

$$
\mathcal{W}(\lambda)=\left.i W[\varphi]\right|_{\varphi_{c}(t)=e V t, \varphi_{\Delta}(t)=\lambda \theta\left(t_{0} / 2+t\right) \theta\left(t_{0} / 2-t\right)},
$$

where $\theta(t)$ is the step function. Then the generating functional is reduced to the cummulant generating function (CGF) for the number of transmitted electrons $q$ during a measurement, $\mathcal{W}(\lambda)=\sum_{n=0}^{\infty}\left\langle\left\langle\delta q^{n}\right\rangle\right\rangle(i \lambda)^{n} / n$ !. The distribution of $q$ (equivalently current $I \equiv e q / t_{0}$ ) is given by the inverse Fourier transformation,

$$
P=\frac{1}{2 \pi} \int_{-\pi}^{\pi} d \lambda \mathrm{e}^{\mathcal{W}(\lambda)-i q \lambda} \approx \mathrm{e}^{\mathcal{W}\left(\lambda^{*}\right)-i\left(t_{0} I / e\right) \lambda^{*}} .
$$

The righthand side is a saddle point solution and $\lambda^{*}$ is obtained by solving $I=-i e \partial_{\lambda} \mathcal{W}\left(\lambda^{*}\right) / t_{0}$ [7]. The approximation is valid for long measurement time since $\mathcal{W}$ is proportional to $t_{0}$ as we will mention later.

We proceed following Ref. [13], where we developed a conserving approximation for second cummulants, i.e. 'noise', based on the Schwinger-Keldysh approach and the Majorana fermion representation. We trace out electron degrees of freedom and obtain an effective action for $c$ and $d$ fields $S^{\lambda} \equiv S_{\mathrm{ch}}+S_{t}^{\lambda}$ consisting of parts describing the charging energy $S_{\mathrm{ch}}$ and the tunneling $S_{t}^{\lambda}$ [14] as

$$
\begin{array}{r}
S_{\mathrm{ch}}=\int_{C} d t\left\{c(t)^{*}\left(i \partial_{t}-\Delta_{0}\right) c(t)+i d(t)^{*} \partial_{t} d(t)\right\}, \\
S_{t}^{\lambda}=-\int_{C} d t d t^{\prime} c^{*}(t) \phi(t) \alpha^{\lambda}\left(t, t^{\prime}\right) \phi\left(t^{\prime}\right) c\left(t^{\prime}\right)+O\left(T_{\mathrm{r}}^{4}\right) .
\end{array}
$$

The term $O\left(T_{\mathrm{r}}^{4}\right)$ describes a phase coherent propagation of an electron between the left and right electrodes and can be neglected for $N_{\mathrm{ch}} \gg 1$. Here $\alpha^{\lambda}=\alpha_{\mathrm{L}}^{\lambda}+\alpha_{\mathrm{R}}^{\lambda}$ is a particle-hole (p-h) GF describing tunneling of an electron from one of the electrodes into the island. In the Keldysh space, the p-h GF is expressed as a $2 \times 2$ matrix rotated by $\lambda_{\mathrm{r}}=\kappa_{\mathrm{r}} \lambda$ along the $x$-axis as

$$
\tilde{\alpha}_{\mathrm{r}}^{\lambda}(\omega)=U_{\lambda_{\mathrm{r}}}^{\dagger} \tilde{\alpha}_{\mathrm{r}}(\omega) U_{\lambda_{\mathrm{r}}}, \tilde{\alpha}_{\mathrm{r}}(\omega)=\left(\begin{array}{cc}
0 & \alpha_{\mathrm{r}}^{A}(\omega) \\
\alpha_{\mathrm{r}}^{R}(\omega) & \alpha_{\mathrm{r}}^{K}(\omega)
\end{array}\right),
$$

where $U_{\lambda_{\mathrm{r}}}=\exp \left(-i \lambda_{\mathrm{r}} \boldsymbol{\tau}_{1} / 2\right)$ and $\left[\boldsymbol{\tau}_{1}\right]_{i j}=1-\delta_{i j}$. Retarded and advanced components are given by $\alpha_{\mathrm{r}}^{R}(\omega)=$ $\alpha_{\mathrm{r}}^{A}(\omega)^{*}=-i \pi \alpha_{0}^{\mathrm{r}}\left(\omega-\mu_{\mathrm{r}}\right) E_{C}^{2} /\left\{\left(\omega-\mu_{\mathrm{r}}\right)^{2}+E_{C}^{2}\right\}$, where $\alpha_{0}^{\mathrm{r}} \equiv$ $R_{\mathrm{K}} /\left(4 \pi^{2} R_{\mathrm{r}}\right)$ and we introduced the Lorentzian cutoff to regularize the ultraviolet divergence. A Keldysh component $\alpha_{\mathrm{r}}^{K}(\omega)=2 \alpha_{\mathrm{r}}^{R}(\omega)\left\{n^{+}\left(\omega-\mu_{\mathrm{r}}\right)+n^{-}\left(\omega-\mu_{\mathrm{r}}\right)\right\}$ contains the Bose distribution function $n^{+}(\omega)=1 /\left(\mathrm{e}^{\omega / T}-1\right)$ and $n^{-}=1+n^{+}$.

We observe that only the Dirac fermion $\hat{c}$ may carry physical meaning and its free retarded GF $g_{c}^{R}(\omega)=1 /(\omega+$ $\left.i \eta-\Delta_{0}\right)$ describes dynamics of charge-state excitation $(\eta$ is a positive infinitesimal). Then the self-energy $\Sigma_{c}^{\lambda}=$ $\Sigma_{\mathrm{L}}^{\lambda}+\Sigma_{\mathrm{R}}^{\lambda}$ would account for quantum charge-fluctuations caused by tunneling. The first order expansion in $\alpha_{0}$ is $\Sigma_{\mathrm{r}}^{\lambda}\left(t, t^{\prime}\right)=-i g_{\phi}\left(t^{\prime}, t\right) \alpha_{\mathrm{r}}^{\lambda}\left(t, t^{\prime}\right)$, where $g_{\phi}$ is the $d$-field GF. Keldysh and retarded components of the self-energy for zero counting-filed $\tilde{\Sigma}_{\mathrm{r}}(\omega)$ are given by

$$
\Sigma_{\mathrm{r}}^{K}(\omega)=2 \alpha_{\mathrm{r}}^{R}(\omega), \quad \Sigma_{\mathrm{r}}^{R}(\omega)=\int d \omega^{\prime} \frac{i \alpha_{\mathrm{r}}^{K}\left(\omega^{\prime}\right)}{(2 \pi)\left(\omega+i \eta-\omega^{\prime}\right)} .
$$

The integration results in a logarithm as $\Sigma_{c}^{R}(\omega) \approx$ $\alpha_{0} \ln \left\{2 E_{C} /(e V)\right\} \omega-i \Gamma / 2$ for $|\omega| \ll e V \quad(T=0$ and a symmetric case, i.e. $R_{\mathrm{L}}=R_{\mathrm{R}}$ and $C_{\mathrm{L}}=C_{\mathrm{R}}$ ). The imaginary part describes the lifetime broadening of chargestate level caused by dissipative current: $\Gamma$ is the sum of tunneling rates $\Gamma=\Gamma_{\mathrm{IL}}+\Gamma_{\mathrm{LI}}+\Gamma_{\mathrm{IR}}+\Gamma_{\mathrm{RI}}$, where $\Gamma_{\mathrm{rI} / \mathrm{Ir}}=$ $\left(\Delta_{0}-\mu_{\mathrm{r}}\right) n^{ \pm}\left(\Delta_{0}-\mu_{\mathrm{r}}\right) /\left(e^{2} R_{\mathrm{r}}\right)$ is the tunneling rate into (out of) the island through the junction $r$ calculated within Fermi's golden rule.

We can perform a systematic perturbative expansion in $\alpha_{0}$ for the CGF using diagrams 13]. For example, the first order expansion is given by $\mathcal{W}^{[1]}(\lambda)=$ $-\int_{C} d t d t^{\prime} g_{c}\left(t, t^{\prime}\right) \Sigma_{c}^{\lambda}\left(t^{\prime}, t\right)$. We proceed by projecting the time on $C$ onto the real axis. For enough long measurement time, we can approximate an integral $\delta_{t_{0}}(\omega) \equiv$ $\int_{-t_{0} / 2}^{t_{0} / 2} d t \mathrm{e}^{-i \omega t} /(2 \pi)$ and its square normalized by $t_{0}$, $2 \pi \delta_{t_{0}}(\omega)^{2} / t_{0}$, as the $\delta$-function $\delta(\omega)$. Especially, the latter equation ensures that $\mathcal{W}$ is proportional $t_{0}$. By performing the Fourier transformation, we obtain

$$
\begin{aligned}
& \mathcal{W}^{[1]}(\lambda) \approx-t_{0} \int d \omega \operatorname{Tr}\left\{\tilde{g}_{c}(\omega) \tau_{1} \tilde{\Sigma}_{c}^{\lambda}(\omega) \tau_{1}\right\} /(2 \pi) \\
= & t_{0} \sum_{\mathrm{r}=\mathrm{L}, \mathrm{R}}\left\{P_{-} \Gamma_{\mathrm{rI}}\left(\mathrm{e}^{i \lambda_{\mathrm{r}}}-1\right)+P_{+} \Gamma_{\mathrm{Ir}}\left(\mathrm{e}^{-i \lambda_{\mathrm{r}}}-1\right)\right\},
\end{aligned}
$$

where we utilized the expression of Keldysh component for free $c$-field GF $g_{c}^{K}(\omega)=2 i \operatorname{Im} g_{c}^{R}(\omega)\left(P_{-}-P_{+}\right)$. It contains initial distribution probabilities of charge state 
$P_{ \pm}=1 /\left(\mathrm{e}^{ \pm \Delta_{0} / T}+1\right)$. Equation (9) is the sum of CGFs of Poissonian distribution describing tunneling of an electron into and out of the island. Here, we observe that the naive first order expansion Eq. (9) is insufficient: First, it includes the initial distribution probabilities, which should be replaced with stationary distribution probabilities. Second, the CGF should depend only on $\lambda_{\mathrm{L}}-\lambda_{\mathrm{R}}=\lambda$ from the charge conservation [15]. Moreover, the first order expansion is obviously insufficient to reproduce the logarithmic behavior of the scaling analysis.

Above flaws can be removed by accounting for a subclass of diagrams. Practically, we simply sum up the geometric series in $\left(\tilde{g}_{c} \boldsymbol{\tau}_{1} \tilde{\Sigma}_{c}^{\lambda} \boldsymbol{\tau}_{1}\right)$, which contains leading logarithms, i.e. powers of $\alpha_{0} \ln \left\{2 E_{C} /(e V)\right\}$ :

$$
\begin{gathered}
\mathcal{W}(\lambda) \approx \frac{t_{0}}{2 \pi} \int d \omega \operatorname{Tr} \ln \left[\tilde{g}_{c}(\omega)^{-1}-\tau_{1} \tilde{\Sigma}_{c}^{\lambda}(\omega) \tau_{1}\right] \\
=\frac{t_{0}}{2 \pi} \int d \omega \ln \left\{1+T^{F}(\omega) f^{+}\left(\omega-\mu_{\mathrm{L}}\right) f^{-}\left(\omega-\mu_{\mathrm{R}}\right)\left(\mathrm{e}^{i \lambda}-1\right)\right. \\
\left.+T^{F}(\omega) f^{+}\left(\omega-\mu_{\mathrm{R}}\right) f^{-}\left(\omega-\mu_{\mathrm{L}}\right)\left(\mathrm{e}^{-i \lambda}-1\right)\right\}+O(\eta),(10)
\end{gathered}
$$

where $f^{-}=1-f^{+}$and we removed a constant to fulfill the normalization condition $\mathcal{W}(0)=0$. Now $P_{ \pm}$is absorbed in $O(\eta)$ and only $\lambda$ appears in the expression. Equation (10) looks similar to the Levitov-Lesovik formula [16]. But for our case the effective transmission probability $T^{F}(\omega)=-\alpha_{\mathrm{L}}^{K}(\omega) \alpha_{\mathrm{R}}^{K}(\omega) /\left|\omega-\Delta_{0}-\Sigma_{c}^{R}(\omega)\right|^{2}$ accounts for strong quantum charge-fluctuations.

In fact, Eq. (10) is reduction of an approximate Keldysh generating functional Eq. (25) in Ref. 13 to the CGF. Therefore, first and second cummulants, $\langle I\rangle=$ $e\langle\langle\delta q\rangle\rangle / t_{0}$ and $S_{I I}=2 e^{2}\left\langle\left\langle\delta q^{2}\right\rangle\right\rangle / t_{0}$, reproduce an average current and a zero-frequency noise in Ref. 13]. Especially, for the average current, our approximation and the 'resonant tunneling approximation' [9] are equivalent.

Limiting cases. - In the limit of $\alpha_{0} \rightarrow 0$, we confirm that Eq. (10) reproduces the 'orthodox' theory [7]:

$$
\begin{array}{r}
\mathcal{W}^{(1)}(\lambda)=t_{0} \Gamma(\sqrt{D(\lambda)}-1) / 2, \\
D(\lambda)=1+4 \frac{\Gamma_{\mathrm{LI}} \Gamma_{\mathrm{IR}}}{\Gamma^{2}}\left(\mathrm{e}^{i \lambda}-1\right)+4 \frac{\Gamma_{\mathrm{RI}} \Gamma_{\mathrm{IL}}}{\Gamma^{2}}\left(\mathrm{e}^{-i \lambda}-1\right) .
\end{array}
$$

It is noticed that though $\mathcal{W}^{(1)}$ is proportional to $\alpha_{0}$, it is different from the naive first order expansion $\mathcal{W}^{[1]}$. The second order expansion in $\alpha_{0}$ reads,

$$
\mathcal{W}^{(2)}(\lambda)=\mathcal{W}^{\cot }(\lambda)+\partial_{\Delta_{0}}\left\{\operatorname{Re} \Sigma_{c}^{R}\left(\Delta_{0}\right) \mathcal{W}^{(1)}(\lambda)\right\} .
$$

The second term provides the renormalization of the system parameters up to first order in $\alpha_{0}$ [17. The first term is the CGF of a bidirectional Poissonian process

$$
\mathcal{W}^{\cot }(\lambda)=t_{0}\left\{\gamma^{+}\left(\mathrm{e}^{i \lambda}-1\right)+\gamma^{-}\left(\mathrm{e}^{-i \lambda}-1\right)\right\},
$$

governed by the cotunneling rate, $\gamma^{ \pm}=2 \pi \alpha_{0}^{\mathrm{L}} \alpha_{0}^{\mathrm{R}} \int d \omega(\omega-$ $\left.\mu_{\mathrm{L}}\right)\left(\omega-\mu_{\mathrm{R}}\right) n^{ \pm}\left(\omega-\mu_{\mathrm{L}}\right) n^{\mp}\left(\omega-\mu_{\mathrm{R}}\right) \operatorname{Re}\left\{\left(\omega+i \eta-\Delta_{0}\right)^{-2}\right\}$. The first term is relevant in the $\mathrm{CB}$ regime and is consistent with the FCS theory of quasiparticle tunneling [18]. Since a tunneling quasiparticle is an electron tunneling from the left electrode to the right electrode with a p-h pair left in the island, the tunneling rate is not proportional to $\left|T_{\mathrm{L} / \mathrm{R}}\right|^{2}$ but $\left|T_{\mathrm{L}} T_{\mathrm{R}}\right|^{2}$.

We will discuss the saddle point solution of the current distribution $P$, Eq. (4). Solid lines in Fig. 1(a) show the logarithm of nonequilibrium distribution for the symmetric case at $T=0$. The conductance is small and thus $\mathcal{W}^{(1)}$ and $\mathcal{W}^{\text {cot }}$ well approximate CGFs deep in the ST regime and in the $\mathrm{CB}$ regime, respectively. At $\Delta_{0}=0$, the CGF is $\mathcal{W}^{(1)}(\lambda) \approx 2 \bar{q}\left(\mathrm{e}^{i \lambda / 2}-1\right)$, where $e \bar{q} / t_{0}=G_{0} V / 2$ and $1 / G_{0}=R_{\mathrm{L}}+R_{\mathrm{R}}$. The factor $\mathrm{e}^{i \lambda / 2}$ results in a sub-Poissonian value of the Fano factor $S_{I I} /(2 e\langle I\rangle) \approx 1 / 2$ indicating that tunneling processes are correlated. The meaning of the 'correlated process' can be understood from an explicit form of distribution $P(q)=\sum_{q_{\mathrm{L}}, q_{\mathrm{R}}=0}^{\infty} P_{\mathrm{P}}\left(q_{\mathrm{L}}\right) P_{\mathrm{P}}\left(q_{\mathrm{R}}\right) \delta_{q,\left(q_{\mathrm{L}}+q_{\mathrm{R}}\right) / 2}$ obtained by simply performing the inverse Fourier transformation Eq. (4) without the saddle point approximation. For symmetric case, numbers of transmitted electrons through the junctions $\mathrm{L}$ and $\mathrm{R}, q_{\mathrm{L}}$ and $q_{\mathrm{R}}$, follow the same Poissonian distribution $P_{\mathrm{P}}(q)=\bar{q}^{q} \mathrm{e}^{-\bar{q}} / q$ !. The Kronecker delta implies that $q_{\mathrm{L}}$ and $q_{\mathrm{R}}$ are not independent variables.

As $\Delta_{0}$ increases and approaches a threshold value $\Delta_{0}=$ $\mathrm{eV} / 2$, the left junction becomes dominant $\mathcal{W}^{(1)}(\lambda) \approx$ $t_{0} \Gamma_{\mathrm{LI}}\left(\mathrm{e}^{i \lambda}-1\right)$ and the Poissonian value is approached $S_{I I} /(2 e\langle I\rangle) \approx 1$. The Fano factor continues to be unity in the $\mathrm{CB}$ regime, because the CGF is also that of the Poissonian distribution $\mathcal{W}^{\text {cot }}$. However, since tunneling quasiparticles are different in two regimes, near the threshold, there is a regime where neither the 'orthodox' theory $\mathcal{W}^{(1)}$ nor the 'cotunneling theory' $\mathcal{W}^{\text {cot }}$ does not work. The plot for $\Delta_{0} / e V=0.475$ in Fig. 1(a) is such an example. The 'orthodox' theory [dashed line] cannot fit the solid line.

Renormalization and lifetime broadening effects. As the conductance increases, the quantum chargefluctuations are promoted. However, when the lifetime broadening is negligible, $z_{0} \Gamma \ll e V[\Lambda=$ $\left.\max \left(\left|z_{0} \Delta_{0}\right|, 2 \pi T,|e V| / 2\right)\right]$, the 'orthodox' theory $\mathcal{W}^{(1)}$ with renormalized parameters $z_{0} \alpha_{0}$ and $z_{0} \Delta_{0}$ is valid. Figure 1(b-1), the current distribution at $\Delta_{0}=0$ and a small bias voltage, indicates the renormalization effect: Since $z_{0}$ decreases with increasing $\alpha_{0}$, the mean value, i.e. a peak position, shifts leftwards. The above scenario could fail in a regime $T_{\mathrm{K}}=E_{C} \mathrm{e}^{-1 /\left(2 \alpha_{0}\right)} /(2 \pi) \gg \Lambda$ where leading logarithms would be not enough.

If we replot Fig. 1(b-1) with vertical and horizontal axes normalized not by $G_{0} V / 2$ but by $\langle I\rangle$, three curves almost overlap (not shown), i.e. the renormalization factor is normalized away. A qualitative change occurs when the bias voltage increases and the lifetime broadening effect is enhanced [Fig. 1(b-2)]. We observe a shrinking of the distribution with increasing $\alpha_{0}$. The shrinking 

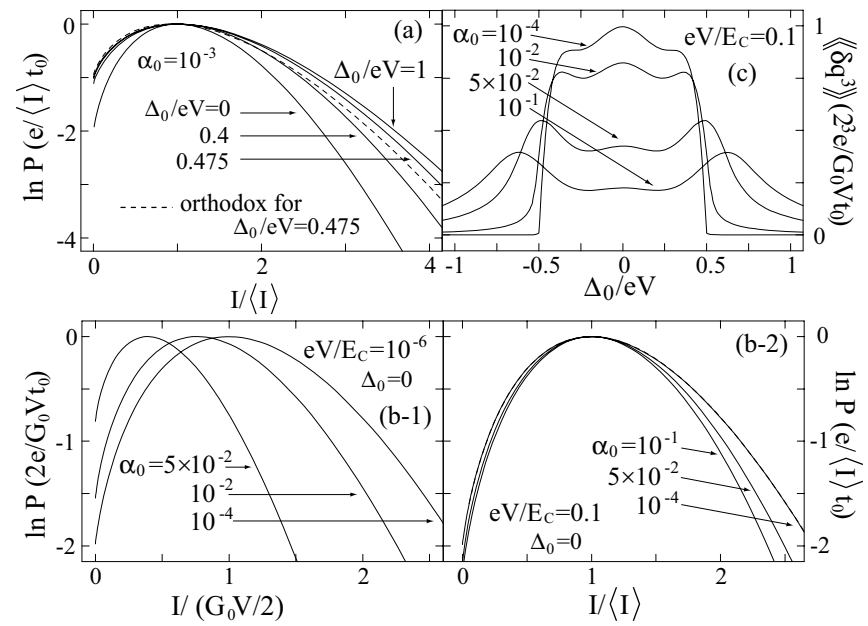

FIG. 1: The zero-temperature current distribution for $R_{\mathrm{L}}=$ $R_{\mathrm{R}}$ and $C_{\mathrm{L}}=C_{\mathrm{R}}[(\mathrm{a}),(\mathrm{b}-1,2)]$. (a) Solid lines are plots for various excitation energies for small conductance. A dashed line is a result of the 'orthodox' theory at $\Delta_{0} /(\mathrm{eV})=0.475$. Plots at $\Delta_{0}=0$ for various conductances with small (b-1) and large (b-2) bias voltages. Axes are normalized by the average current $\langle I\rangle$ (b-2) and that of the 'orthodox' theory $G_{0} V / 2$ (b1). (c) The skewness at $e V=0.1 E_{C}$ for various conductances.

is compatible with the suppression of the Fano factor predicted in Ref. 13]. The FCS analysis gives more information beyond the noise analysis. From the current distribution, we can see the probability of relatively large current is suppressed.

Let us discuss the lifetime broadening effect for symmetric case more quantitatively. For $\mathrm{eV} \gg T_{\mathrm{K}}$, the real part of $\Sigma_{c}^{R}$ can be neglected and only the imaginary part describing the lifetime broadening is important, $\Sigma_{c}^{R}(\omega) \approx-i \pi \alpha_{0} e V$. Then the CGF at $\Delta_{0}=0$ is estimated as

$$
\begin{aligned}
\mathcal{W}(\lambda) & \approx 2 \bar{q}\left\{\left(\mathrm{e}^{i \lambda / 2}-1\right)-2 \alpha_{0}\left(\mathrm{e}^{i \lambda}-1\right)\right. \\
& \left.+\pi^{2} \alpha_{0}^{2}\left(\mathrm{e}^{i 3 \lambda / 2}-\mathrm{e}^{i \lambda / 2}\right) / 2+O\left(\alpha_{0}^{3}\right)\right\}
\end{aligned}
$$

and the 'Fano factor' reads $\left\langle\left\langle\delta q^{n}\right\rangle\right\rangle /\langle\langle\delta q\rangle\rangle=2^{1-n}\{1-$ $\left.4 \alpha_{0}\left(2^{n-1}-1\right)+O\left(\alpha_{0}^{2}\right)\right\}$. We can see higher cummulants are suppressed as $\alpha_{0}$ increases. Figure 1(c) shows the skewness $\left\langle\left\langle\delta q^{3}\right\rangle\right\rangle$ as a function of $\Delta_{0}$. A double-peak structure growing with increasing the conductance is obtained. Such a characteristic structure might be measurable for a present-day experiment [19].

Summing up, we evaluated the FCS of SET. The strong nonequilibrium quantum charge-fluctuations are accounted for by summing up a subclass of diagrams including leading logarithms. Our approximation reproduces the 'orthodox' theory in the limit of $\alpha_{0} \rightarrow 0$ and gives the bidirectional Poissonian distribution governed by the cotunneling rate in the $\mathrm{CB}$ regime. Nonequilibrium quantum charge-fluctuations induce not only the logarithmic renormalization of the conductance and the charging energy but also the lifetime broadening of the charge-state level. It may suppress the probability of relatively large current and higher cummulants of current fluctuations.

We would like to thank D. Bagrets, A. Braggio, Y. Gefen, D. Golubev, J. König, G. Schön and A. Shnirman for valuable discussions. Y.U. was supported by the DFG-Forschungszentrum CFN.

[1] Quantum Noise in Mesoscopic Physics, Vol. 97 of NATO Science Series II: Mathematics, Physics and Chemistry edited by $\mathrm{Yu}$. V. Nazarov (Kluwer Academic Publishers, Dordrecht/Boston/London, 2003).

[2] I. Safi, and H. Saleur, Phys. Rev. Lett. 93, 126602 (2004).

[3] M. Kindermann, and B. Trauzettel, cond-mat/0408666

[4] A. Komnik, and A. O. Gogolin, cond-mat/0411019

[5] D. S. Golubev, J. König, H. Schoeller, G. Schön and A. D. Zaikin, Phys. Rev. B 56, 15782 (1997) and references therein.

[6] K. A. Matveev, Sov. Phys. JETP. 72, 892 (1991).

[7] D. A. Bagrets, and Yu. V. Nazarov, Phys. Rev. B 67, 085316 (2003).

[8] A complete version covering also the strong tunneling regime $R_{\mathrm{K}} \gtrsim R_{\mathrm{T}}$ will be published elsewhere.

[9] H. Schoeller, and G. Schön, Phys. Rev. B 50, 18436 (1994).

[10] Y. Isawa, and H. Horii, J. Phys. Soc. Japan 69, 655 (2000); H. J. Spencer, and S. Doniach, Phys. Rev. Lett. 18, 994 (1967).

[11] K.-C. Chou, Z.-B. Su, B.-L. Hao and L. Yu, Phys. Rep. 118, 1 (1985); A. Kamenev, cond-mat/0412296

[12] A. Kamenev, cond-mat/0109316

[13] Y. Utsumi, H. Imamura, M. Hayashi and H. Ebisawa, Phys. Rev. B 67, 035317 (2003).

[14] The Majorana fermion representation induces the $Z_{2}$ gauge symmetry under the transformation $\phi \rightarrow-\phi$, $c \rightarrow-c$ and $c^{*} \rightarrow-c^{*}$, [P. Coleman, L. B. Ioffe, and A. M. Tsvelik, Phys. Rev. B 52, 6611 (1995)]. For practical calculations, one must be careful to fix gauge [A. Shnirman, and Yu. Makhlin, Phys. Rev. Lett. 91, 207204 (2003)].

[15] W. Belzig, cond-mat/0312180

[16] L. S. Levitov, and G. B. Lesovik, JETP lett. 58, 230 (1993).

[17] The system parameters $\alpha_{0}$ and $\Delta_{0}$ are renormalized as $\alpha_{0}\left\{1+\partial_{\Delta_{0}} \operatorname{Re} \Sigma_{c}^{R}\left(\Delta_{0}\right)\right\}$ and $\Delta_{0}+\operatorname{Re} \Sigma_{c}^{R}\left(\Delta_{0}\right)$ in the same way as for the average current [J. König, H. Schoeller, and G. Schön, Phys. Rev. Lett. 78, 4482 (1997) ;Phys. Rev. B 58, 7882 (1998)]. Equation (13) is consistent with the recent systematic second-order perturbation theory based on the real-time diagrammatic technique [A. Braggio, J. König, and R. Fazio, unpublished].

[18] L. S. Levitov, and M. Reznikov, Phys. Rev. B 70, 115305 (2004).

[19] B. Reulet, J. Senzier and D. E. Prober, Phys. Rev. Lett. 91, 196601 (2003). 